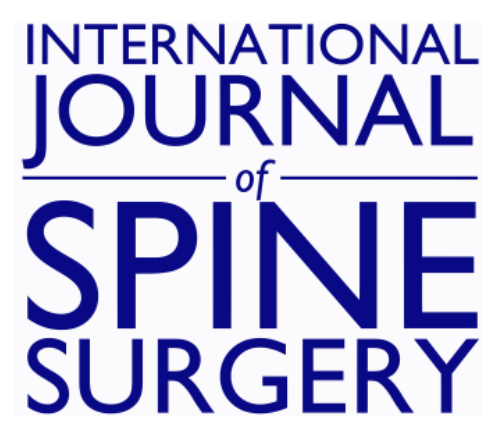

\title{
A Simpler, Modified Frailty Index Weighted by Complication Occurrence Correlates to Pain and Disability for Adult Spinal Deformity Patients
}

PETER G. PASSIAS, COLE A. BORTZ, KATHERINE E. PIERCE, HADDY ALAS, AVERY
BROWN, DENNIS VASQUEZ-MONTES, SARA NAESSIG, WALEED AHMAD, BASSEL G.
DIEBO, TINA RAMAN, THEMISTOCLES S. PROTOPSALTIS, AARON J. BUCKLAND,
MICHAEL C. GERLING, RENAUD LAFAGE and VIRGINIE LAFAGE

Int J Spine Surg 2020, 14 (6) 1031-1036

doi: https://doi.org/10.14444/7154

http://ijssurgery.com/content/14/6/1031

This information is current as of April 26, 2023.

Email Alerts Receive free email-alerts when new articles cite this article. Sign up at: http://ijssurgery.com/alerts 


\title{
A Simpler, Modified Frailty Index Weighted by Complication Occurrence Correlates to Pain and Disability for Adult Spinal Deformity Patients
}

\author{
PETER G. PASSIAS, MD,${ }^{1}$ COLE A. BORTZ, BA, ${ }^{1}$ KATHERINE E. PIERCE, BS, ${ }^{1}$ HADDY ALAS, BS, ${ }^{1}$ \\ AVERY BROWN, BS, ${ }^{1}$ DENNIS VASQUEZ-MONTES, MS,${ }^{1}$ SARA NAESSIG, BS, ${ }^{1}$ WALEED AHMAD, MS, ${ }^{1}$ \\ BASSEL G. DIEBO, MD, ${ }^{2}$ TINA RAMAN, MD,${ }^{1}$ THEMISTOCLES S. PROTOPSALTIS, MD,${ }^{1}$ AARON J. \\ BUCKLAND, MBBS, FRACS, ${ }^{1}$ MICHAEL C. GERLING, MD, ${ }^{1}$ RENAUD LAFAGE, MS, ${ }^{3}$ VIRGINIE \\ LAFAGE, PHD $^{3}$ \\ ${ }^{I}$ Department of Orthopedics, NYU Langone Orthopedic Hospital, New York, New York, ${ }^{2}$ Department of Orthopedics, SUNY Downstate Medical Center, \\ Brooklyn, New York, ${ }^{3}$ Department of Orthopedics, Hospital for Special Surgery, New York, New York
}

\begin{abstract}
Background: The Miller et al adult spinal deformity frailty index (ASD-FI) correlates with complication risk; however, its development was not rooted in clinical outcomes, and the 40 factors needed for its calculation limit the index's clinical utility. The present study aimed to develop a simplified, weighted frailty index for ASD patients

Methods: This study is a retrospective review of a single-center database. Component ASD-FI parameters contributing to overall ASD-FI score were assessed via Pearson correlation. Top significant, clinically relevant factors were regressed against ASD-FI score to generate the modified ASD-FI (mASD-FI). Component mASD-FI factors were regressed against incidence of medical complications, and factor weights were calculated from regression of these coefficients. Total mASD-FI score ranged from 0 to 21 , and was calculated by summing weights of expressed parameters. Linear regression and published ASD-FI cutoffs generated corresponding mASD-FI frailty cutoffs: not frail $(\mathrm{NF},<7)$, frail $(7-12)$, severely frail $(\mathrm{SF},>12)$. Analysis of variance assessed the relationship between frailty category and validated baseline measures of pain and disability at baseline.

Results: The study included 50 ASD patients. Eight factors were included in the mASD-FI. Overall mean mASDFI score was $5.7 \pm 5.2$. Combined, factors comprising the mASD-FI showed a trend of predicting the incidence of medical complications (Nagelkerke $R^{2}=0.558$; Cox \& Snell $R^{2}=0.399 ; P=.065$ ). Breakdown by frailty category is NF $(70 \%)$, frail $(12 \%)$, and SF $(18 \%)$. Increasing frailty category was associated with significant impairments in measures of pain and disability: Oswestry Disability Index (NF: 23.4; frail: 45.0; SF: 49.3; $P<.001$ ), SRS-22r (NF: 3.5; frail: 2.6; SF: $2.4 ; P=.001$ ), Pain Catastrophizing Scale (NF: 41.9; frail: 32.4; SF: 27.6; $P<.001$ ), and NRS Leg Pain (NF: 2.3 ; frail: 7.2 ; SF: $5.6 ; P=.001)$.

Conclusions: This study modifies an existing ASD frailty index and proposes a weighted, shorter mASD-FI. The mASD-FI relies less on patient-reported variables, and it weights component factors by their contribution to adverse outcomes. Because increasing mASD-FI score is associated with inferior clinical measures of pain and disability, the mASD-FI may serve as a valuable tool for preoperative risk assessment.
\end{abstract}

Other \& Special Categories

Keywords: adult spinal deformity, Frailty Index, Modified Frailty Index, ASD

\section{INTRODUCTION}

Frailty, or vulnerability to adverse health outcomes, is gaining recognition as a key consideration in preoperative risk stratification and patient counseling. To quantify frailty, some clinicians have adopted a "deficit accumulation"-based approach, developing frailty indices that tally combinations of disabilities, comorbidities, and social situations associated with physiologic decline. ${ }^{1,2}$ These frailty screening tools are validated in a number of patient populations and have been used in the adult spinal deformity (ASD) literature to identify patients at risk for adverse outcomes following deformitycorrective surgery. ${ }^{3-5}$

Although there is no established consensus for detecting frailty in ASD patients, one of the most commonly cited ASD-specific frailty assessment tools is the 2017 Miller et $\mathrm{al}^{6}$ ASD frailty index (ASD-FI). Developed in a prospectively collected population of 450 ASD patients, the ASD-FI 
includes 40 distinct health deficits assessing physical disability, medical comorbidities, activities of daily living, mood, and cognition. A patient's ASD-FI score is generated by calculating the mean of expressed deficits, yielding a score from 0 to 1 , with higher values indicating greater frailty. The ASD-FI has been externally validated in multiple databases, and it has been shown to correlate with length of hospital stay, incidence of proximal junctional kyphosis, risk of major complication, and risk of reoperation. ${ }^{6-8}$

Despite the ASD-FI's validity as a risk assessment tool, its calculation requires a complete set of 40 variables, making it cumbersome to use in a clinical setting. Additionally, more than half of the 40 health deficits included in the ASD-FI stem from patient-reported outcome measures - some of which are not always available in a patient's chart or medical record. To address these problems, this study aims to crease a simpler, modified ASD frailty index (mASD-FI) that relies less on patientreported outcomes. Instead of simply calculating the mean of all expressed frailty index health deficits, this study weights the component deficits by their overall contribution to the risk of adverse outcomes.

\section{MATERIALS AND METHODS}

\section{Study Design}

This study is a retrospective analysis of consecutive adult spinal deformity (ASD) patients. Each institution obtained approval from its local Institutional Review Board to enroll patients in the prospective database, and informed consent was obtained from each patient. All patients were enrolled at a single spine center from May 2009 to February 2012, following study approval by the Institutional Review Board. Patients provided informed consent prior to enrollment. Patients eligible for study enrollment were older than 18 years seeking either operative or nonoperative treatment for ASD, defined radiographically as baseline scoliosis $>20^{\circ}$ (measured by major coronal Cobb angle), sagittal vertical axis $\geq 5 \mathrm{~cm}$, pelvic tilt $\geq 25^{\circ}$, and/or thoracic kyphosis $>60^{\circ}$. Patients included in this analysis also had complete data for all the component health deficits in the modified ASD frailty index (mASD-FI).

\section{Development of the mASD-FI}

This study modifies the 40-factor ASD-FI published by Miller et al in 2017. ${ }^{6}$ For each patient, ASD-FI score was calculated. Pearson bivariate correlation then assessed the relationships between each of the 40 component health deficits and overall ASD-FI score. The top statistically significant, clinically relevant health deficits identified in the above correlation analysis were included in a multiple linear regression model predicting overall ASD-FI score. This multiple linear regression model was limited to 8 independent health deficits to minimize model saturation and overfitting. ${ }^{9}$ The 8 factors comprising this statistically significant model were ultimately included in the mASD-FI.

\section{Weighting and Calculation of the mASD-FI}

Component factors comprising the mASD-FI were weighted differently by their relationship to risk of medical complications. Briefly, factors comprising the mASD-FI were regressed against the incidence of medical complications in a multiple linear regression model. Variance inflation factors from this regression model were used to assess harmful multicolinearity across the model's independent variables, defining harmful multicolinearity as variance inflation factors $>10 .{ }^{10}$ Because no harmful multicolinearity was detected, a regression $\beta$ coefficient-based scoring system was used to assign weights to each independent mASD-FI variable. As published, the absolute values of regression beta coefficients were divided by the smallest value of regression coefficient, and then rounded to the nearest integer, yielding a weight for each independent mASD-FI factor. ${ }^{11,12}$ Patient mASD-FI score was calculated by summing weights of the expressed mASD-FI parameters, resulting in a score ranging from 0 to 21 .

\section{Statistical Analysis}

Linear regression established a relationship between ASD-FI and mASD-FI scores; from this relationship, the published ASD-FI score cutoffs for not frail (NF), frail, and severely frail (SF) patients were used to generate corresponding mASD-FI frailty cutoffs: NF $(<7)$, frail $(7-12)$, and SF $(>12){ }^{6}$ Analysis of variance assessed the relationship between increasing frailty category and a number of factors, including demographics, baseline sagittal alignment, and the following baseline 
Table 1. Baseline radiographic overview of the overall cohort $(N=50)$.

\begin{tabular}{lc}
\hline Radiographic Parameter & Baseline Value \pm SD \\
\hline PI, ${ }^{\circ}$ & $51.3 \pm 13$ \\
PT, ${ }^{\circ}$ & $23.4 \pm 12.4$ \\
PI-LL, ${ }^{\circ}$ & $13.8 \pm 24$ \\
TK, ${ }^{\circ}$ C1 SVA, mm & $-27.7 \pm 19.3$ \\
C7-S1 SVA, mm & $31.7 \pm 78.7$ \\
C2-Slope, ${ }^{\circ}$ & $56.3 \pm 90$ \\
T1 sl-CL, & $23.6 \pm 13.5$ \\
C2-C7 CL, ${ }^{\circ}$ & $16.1 \pm 10.8$ \\
C2-C7 SVA, mm & $8.3 \pm 13$ \\
\end{tabular}

Abbreviations: CL, C2-C7 lordosis; PI, pelvic incidence; PI-LL, pelvic incidence minus lumbar lordosis; PT, pelvic tilt; SVA, sagittal vertical axis; TK, thoracic kyphosis; TS, T1 slope; TS-CL, T1 slope minus C2-C7 lordosis.

measures of health-related quality of life (HRQL): Oswestry Disability Index (ODI), SRS-22r, SF-36 Physical Component score, and Numeric Rating Scales (NRS) for Back and Leg Pain. All analysis was performed using SPSS software (v23.0, Armonk, NY). Statistical significance was set to $P<$ .05 .

\section{RESULTS}

\section{Cohort Overview and mASD-FI Development}

Overall, 50 patients met inclusion criteria for analysis. The included cohort had a mean age of 52 \pm 20 years, a mean body mass index of $26.7 \pm 6.8$ $\mathrm{kg} / \mathrm{m}^{2}$, and comprised $75.9 \%$ women. Table 1 details a baseline radiographic overview of the included cohort, showing moderate lumbopelvic deformity: PI-LL (11.3 \pm 23.0) and pelvic tilt (22.8 \pm 12.6). Together, the 8 health deficits comprising the mASD-FI accounted for $85.0 \%$ of the variance in ASD-FI score. Combined, these factors comprising the mASD-FI showed a trend of predicting the incidence of medical complications (Nagelkerke $R^{2}=0.558 ;$ Cox \& Snell $R^{2}=0.399 ; P=$ .065). This allowed for the development of complication-based weights for mASD-FI component factors (Table 2).
At baseline, mASD-FI scores ranged from 0.0 to 18.0, and the overall cohort had a mean mASD-FI score of $5.1 \pm 4.9$. Overall patient breakdown by mASD-FI frailty category was: NF $(70 \%)$, frail $(12 \%)$, and SF $(18 \%)$.

\section{Baseline Demographics and Alignment by Frailty Status}

Increases in frailty category, as assessed by the mASD-FI, were associated with significant increases in age (NF: $47.0 \pm 20.7$ years; frail: $63.6 \pm 9.6$ years; SF: $63.5 \pm 12.9$ years; $P=.022)$, body mass index $\left(24.2 \pm 3.3 \mathrm{~kg} / \mathrm{m}^{2} ; 27.6 \pm 3.3 \mathrm{~kg} / \mathrm{m}^{2} ; 34.4 \pm\right.$ $\left.3.3 \mathrm{~kg} / \mathrm{m}^{2} ; P<.001\right)$, and Charlson Comorbidity Index score (NF: $0.9 \pm 1.3$; frail: $1.7 \pm 0.8$; SF: 2.4 $\pm 1.9 ; P=.009)$. Table 3 outlines differences in baseline sagittal alignment by increasing frailty category. Although there were no statistically significant differences in alignment across frailty groups, frail and SF patients showed a trend of greater global malalignment, as assessed by sagittal vertical axis, than nonfrail patients $(P=.066)$.

\section{HRQL by Frailty Status}

Increasing frailty category, as assessed by the mASD-FI, was associated with significantly greater levels of baseline pain and disability (Table 4). Specifically, increasing frailty category was associated with a corresponding increase in ODI score and decrease in SRS-22r total score, indicating worse levels of low-back disability and scoliosis HRQL, respectively.

\section{DISCUSSION}

During the last several decades, advances in technology and surgical techniques have allowed surgeons to treat ASD patients with increasingly complex pathologies and comorbidity profiles. ${ }^{13,14}$ Given the increasing heterogeneity of patients

Table 2. Final multiple linear regression model predicting overall patient adult spinal deformity frailty index. ${ }^{a}$

\begin{tabular}{|c|c|c|c|c|}
\hline Variable & Standardized Coefficient $(\beta)$ & Variance Inflation Factor & $\beta$ Weight & $P$ Value \\
\hline $\mathrm{BMI}<18.5$ or $>30 \mathrm{~kg} / \mathrm{m}^{2}$ & -0.015 & 1.21 & 5 & .049 \\
\hline Depression & 0.144 & 1.44 & 5 & .036 \\
\hline Difficulty climbing 1 flight of stairs (SF-36 v2, 3e) & 0.245 & 1.70 & 3 & .001 \\
\hline More than 3 medical comorbidities & 0.157 & 1.48 & 2 & .025 \\
\hline Leg weakness & 0.124 & 1.88 & 2 & .098 \\
\hline Difficulty getting dressed (SF-36 v2, 3j, or LSDI 1 and 2) & 0.373 & 1.52 & 1 & $<.001$ \\
\hline Bladder incontinence & 0.121 & 1.32 & 1 & .064 \\
\hline Deterioration of health within the past year (SF-36 v2, 2) & 0.292 & 1.70 & 1 & $<.001$ \\
\hline
\end{tabular}

Abbreviation: BMI, body mass index.

${ }^{\mathrm{a}} R^{2}=0.850$ 
Table 3. Comparison of baseline radiographic alignment across frailty categories.

\begin{tabular}{|c|c|c|c|c|}
\hline Radiographic Parameter & Not Frail $(n=35)$ & Frail $(n=6)$ & Severely Frail $(n=9)$ & $P$ Value \\
\hline $\mathrm{PI}{ }^{\circ}$ & $50 \pm 14$ & $58.7 \pm 6.6$ & $50.8 \pm 11.5$ & .325 \\
\hline $\mathrm{PT},{ }^{\circ}$ & $22.1 \pm 13.6$ & $26.0 \pm 5.0$ & $27.0 \pm 10.0$ & .533 \\
\hline PI-LL, ${ }^{\circ}$ & $9.3 \pm 25.3$ & $27.0 \pm 15.1$ & $22.8 \pm 18.8$ & .128 \\
\hline TK, ${ }^{\circ}$ & $-30.7 \pm 19.2$ & $-13.5 \pm 8.4$ & $-25.7 \pm 21.8$ & .120 \\
\hline C7-S1 SVA, mm & $15.9 \pm 70.8$ & $92.8 \pm 103.5$ & $57.3 \pm 78.9$ & .066 \\
\hline C2-S1 SVA, mm & $41.8 \pm 79.3$ & $151.6 \pm 148$ & $57.1 \pm 77.3$ & .143 \\
\hline T1 slope, ${ }^{\circ}$ & $22.5 \pm 12.5$ & $23.4 \pm 15.6$ & $27.8 \pm 16.6$ & .590 \\
\hline TS-CL, & $15.6 \pm 10.1$ & $17.3 \pm 17.8$ & $17.4 \pm 12.1$ & .933 \\
\hline 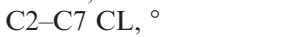 & $7.7 \pm 9.8$ & $8.3 \pm 2.8$ & $10.5 \pm 25.7$ & .917 \\
\hline C2-C7 SVA, mm & $27.9 \pm 16.2$ & $26.3 \pm 19.3$ & $32.7 \pm 19.7$ & .829 \\
\hline
\end{tabular}

Abbreviations: CL, C2-C7 lordosis; PI, pelvic incidence; PI-LL, pelvic incidence minus lumbar lordosis; PT, pelvic tilt; SVA, sagittal vertical axis; TK, thoracic kyphosis; TS, T1 slope; TS-CL, T1 slope minus C2-C7 lordosis.

undergoing ASD-corrective surgery, many studies in the ASD literature emphasize the importance of preoperative risk stratification, or the identification of patients likely to benefit from surgical treatment and patients likely to experience a complication. ${ }^{15-17}$ Within this body of research, a number of studies have demonstrated the importance of frailty in the preoperative risk stratification process. ${ }^{4,18}$ To quantify the frailty status of patients undergoing ASD-corrective surgery, Miller et al developed the ASD-FI, a frailty assessment tool that has since been validated in multiple databases as a predictor of longer hospital stay, complication risk, and risk of reoperation. ${ }^{6-8}$ Despite its validity as a risk stratification tool, the ASD-FI may be impractical to use outside of a research setting because its calculation requires a complete set of 40 variablessome of which rely on multiple HRQL questionnaires not readily available in a patient's medical record. This study proposes a clinically practical, shorter, modified ASD frailty index.

Our results showed that together, 8 health deficits were responsible for $85.0 \%$ of the variation in ASDFI score (Table 2). Because these 8 factors were able to predict a significant proportion of the variation in ASD-FI score, they were ultimately included in our final mASD-FI. Aside from the considerably shorter list of factors needed to calculate mASD-FI score, the mASD-FI constitutes an appreciable deviation from the Miller et al ASD-FI in that its component factors are weighted by their relationship to the incidence of medical complications. Regression coefficient-based weighting of comorbidity scores is common in the risk index literature. ${ }^{19-21} \mathrm{Com}-$ pared with other methodologies, the regression coefficient-based weighting of risk scores has demonstrated superior performance in predicting outcomes. ${ }^{11}$ In weighting the component factors of our frailty index by their relationship to medical complications, we aim to make the mASD-FI more clinically relevant for risk stratification than existing, unweighted frailty indices.

Additionally, whereas calculating the Miller et al ASD-FI requires data from at least 4 separate HRQL questionnaires (SF-36v2, LSDI, SRS-22r, and ODI), calculating mASD-FI score only relies on data from a single questionnaire, the SF-36v2. Multiple studies in the spine literature highlight the administrative burden of HRQL assessments, and longer series of survey questions have been correlated with lower completion rates and lower overall quality of life. ${ }^{22-24}$ Compared with the ASDFI, the mASD-FI offers a lower administrative burden, making its calculation more practical in a clinical setting.

Although the mASD-FI requires fewer variables to calculate than previously published frailty indices, our results suggest that the shortened, 8-factor index is still sufficiently broad to capture a wide range of frailty states. Of a possible 21 points,

Table 4. Comparison of baseline health-related quality-of-life scores across frailty categories.

\begin{tabular}{|c|c|c|c|c|}
\hline Health-Related Quality-Of-Life Assessment & Not Frail $(n=35)$ & Frail $(n=6)$ & Severely Frail $(\mathrm{n}=9)$ & $P$ Value \\
\hline ODI & $23.5 \pm 16.7$ & $45 \pm 20.1$ & $49.3 \pm 16.8$ & $<.001$ \\
\hline SRS-22r total score & $3.5 \pm 0.8$ & $2.6 \pm 0.6$ & $2.4 \pm 0.6$ & .001 \\
\hline SF-36 PCS & $41 \pm 8.6$ & $32.4 \pm 9.9$ & $27.6 \pm 5.3$ & $<.001$ \\
\hline SF-36 MCS & $48.5 \pm 13.5$ & $39 \pm 5.3$ & $36.6 \pm 19.7$ & .050 \\
\hline NRS back pain & $5.7 \pm 3.4$ & $7.8 \pm 2.3$ & $8 \pm 1.7$ & .079 \\
\hline NRS leg pain & $2.3 \pm 3.3$ & $7.2 \pm 3.1$ & $5.6 \pm 2.8$ & .001 \\
\hline
\end{tabular}

Abbreviations: MCS, Mental Component Summary; NRS, Numeric Rating Scale; ODI, Oswestry Disability Index; PCS, Physical Component Summary. 
patient mASD-FI scores in our population spanned from 0 to 18 , with $70 \%$ of patients presenting as NF, $12 \%$ as frail, and $18 \%$ as SF. Although the Miller et al ASD-FI publication reports a slightly smaller proportion of "not frail" patients in their operative ASD population $(41 \% \mathrm{NF}, 35 \%$ frail, $24 \% \mathrm{SF}$ ), this discrepancy may be explained by the fact that our patient population included both operative and nonoperative ASD patients. Although there are few studies in the ASD literature investigating differences in frailty between operative and nonoperative patients, nonoperative ASD patients have been shown to be less comorbid, with significantly lower rates of depression and severe baseline malalignment. $^{25}$

Importantly, our results showed that increasing frailty status, as assessed by the mASD-FI, was associated with higher levels of baseline low back pain and disability, as assessed by both ODI and SRS-22r scores. This finding further suggests that the mASD-FI may serve as a valuable assessment of baseline physiologic decline and vulnerability; however, future studies should investigate the relationship between mASD-FI score and surgical outcomes.

Although weighting mASD-FI variables by their relationship to the incidence of medical complications pegs the frailty index directly to clinical outcomes, this should not lead one to conclude that the factors comprising the mASD-FI are directly related to risk of adverse events. Instead, these factors may be mediating variables that reflect the presence (or absence) of more severe conditions, which in turn may increase the likelihood of an adverse event-this is a key limitation in the interpretation of our frailty index. Our analysis is also limited by small sample size, which reduces the statistical power of our findings. Lastly, although developing the mASD-FI in a population of both operative and nonoperative ASD patients lends our index greater generalizability, additional research is necessary to fully validate the index's utility as a risk stratification tool.

\section{CONCLUSION}

This study modifies an existing ASD frailty index and proposes a weighted, shorter mASD-FI. The mASD-FI relies less on patient-reported variables, and it weights component factors by their contribution to adverse outcomes. Because increasing $\mathrm{mASD}-\mathrm{FI}$ score is associated with inferior clinical measures of pain and disability, the mASD-FI may serve as a valuable tool for preoperative risk assessment.

\section{REFERENCES}

1. Robinson TN, Walston JD, Brummel NE, et al. Frailty for surgeons: review of a national institute on aging conference on frailty for specialists. J Am Coll Surg. 2015;221:1083-1092.

2. Song X, Mitnitski A, Rockwood K. Prevalence and 10year outcomes of frailty in older adults in relation to deficit accumulation. J Am Geriatr Soc. 2010;58:681-687.

3. Buta BJ, Walston JD, Godino JG, et al. Frailty assessment instruments: systematic characterization of the uses and contexts of highly-cited instruments. Ageing Res Rev. 2016;26:53-61.

4. Leven DM, Lee NJ, Kothari P, et al. Frailty index is a significant predictor of complications and mortality after surgery for adult spinal deformity. Spine (Phila Pa 1976). 2016;41:E1394-1401.

5. Kessler RA, De la Garza Ramos R, Purvis TE, et al. Impact of frailty on complications in patients with thoracic and thoracolumbar spinal fracture. Clin Neurol Neurosurg 2018;169:161-165.

6. Miller EK, Neuman BJ, Jain A, et al. An assessment of frailty as a tool for risk stratification in adult spinal deformity surgery. Neurosurg Focus. 2017;43:1-7.

7. Miller EK, Lenke LG, Neuman BJ, et al. External validation of the Adult Spinal Deformity (ASD) Frailty Index (ASD-FI) in the Scoli-RISK-1 patient database. Spine (Phila Pa 1976). 2018;875:61.

8. Miller EK, Vila-Casademunt A, Neuman BJ, et al. External validation of the adult spinal deformity (ASD) frailty index (ASD-FI). Eur Spine J. 2018;43:1-8.

9. McNeish DM. Using lasso for predictor selection and to assuage overfitting: a method long overlooked in behavioral sciences. Multivariate Behav Res. 2015;50:471-484.

10. Alin A. Multicollinearity. Wiley Interdiscip Rev Comput Stat. 2010;2:370-374.

11. Mehta HB, Mehta V, Girman CJ, et al. Regression coefficient-based scoring system should be used to assign weights to the risk index. J Clin Epidemiol. 2016;79:22-28.

12. Sullivan LM, Massaro JM, D'Agostino Sr RB. Presentation of multivariate data for clinical use: The Framingham Study risk score functions. Stat Med. 2004;23:1631-1660.

13. Smith JS, Shaffrey CI, Bess S, et al. Recent and emerging advances in spinal deformity. Neurosurgery. 2017;80:S70-S85.

14. Passias PG, Jalai CM, Worley N, et al. Adult spinal deformity: national trends in the presentation, treatment, and perioperative outcomes from 2003 to 2010. Spine Deform. 2017;5:342-350.

15. Passias PG, Klineberg EO, Jalai CM, et al. Hospital readmission within two years following adult thoracolumbar spinal deformity surgery: prevalence, predictors, and effect on patient-derived outcome measures. Spine (Phila Pa 1976). 2016;41:1-10.

16. Scheer JK, Osorio JA, Smith JS, et al. Development of a preoperative predictive model for reaching the Oswestry Disability Index minimal clinically important difference for adult spinal deformity patients. Spine Deform. 2018;6:593-599. 
17. Scheer JK, Daniels AH, Smith JS, et al. Development of a preoperative predictive model for major complications following adult spinal deformity surgery. J Neurosurg Spine. 2017;1-8.

18. Yagi M, Fujita N, Okada E, et al. Impact of frailty and comorbidities on surgical outcomes and complications in adult spinal disorders. Spine (Phila Pa 1976). 2018;43(18):1259-1267.

19. van Walraven C, Austin PC, Jennings A, et al. A modification of the Elixhauser comorbidity measures into a point system for hospital death using administrative data. Med Care. 2009;47:626-633.

20. Gagne JJ, Glynn RJ, Avorn J, et al. A combined comorbidity score predicted mortality in elderly patients better than existing scores. J Clin Epidemiol. 2011;64:749-759.

21. Mukherjee B, Ou HT, Wang F, et al. A new comorbidity index: the health-related quality of life comorbidity index. $J$ Clin Epidemiol. 2011;64:309-319.

22. Nayak NR, Coats JM, Abdullah KG, et al. Tracking patient-reported outcomes in spinal disorders. Surg Neurol Int. 2015;6:S490-S499.

23. Barton GR, Sach TH, Avery AJ, et al. A comparison of the performance of the EQ-5D and SF-6D for individuals aged $>$ or $=45$ years. Health Econ. 2008;17:815-832.

24. Holland R, Smith RD, Harvey I, et al. Assessing quality of life in the elderly: a direct comparison of the EQ-5D and AQoL. Health Econ. 2004;13:793-805.

25. Smith JS, Lafage V, Shaffrey CI, et al. Outcomes of operative and nonoperative treatment for adult spinal defor- mity (ASD): a prospective, multicenter matched and unmatched cohort assessment with minimum two-year follow-up. Spine $J$. 2014;14(S1):851-861.

Disclosures and COI: No funding was received in relation to the creation of this work. Dr Passias reported personal consulting fees for Spinewave, Zimmer Biomet, DePuy Synthes, and Medicrea outside the submitted work.

Corresponding Author: Peter G. Passias, MD, Division of Spine, Departments of Neurologic and Orthopaedic Surgery, New York Spine Institute, NYU Langone Orthopedic Hospital, 301 East 17th Street, New York, NY 10003. Phone: (516) 357-8777; Fax: (516) 357-0087; E-mail: Peter. Passias@nyumc.org.

Published 22 January 2021

This manuscript is generously published free of charge by ISASS, the International Society for the Advancement of Spine Surgery. Copyright (C) 2020 ISASS. To see more or order reprints or permissions, see http://ijssurgery.com. 